\title{
Understanding Lamin Proteins and Their Roles in Aging and Cardiovascular Diseases
}

Yizhi Jiang and Julie Y. Ji*

Department of Biomedical Engineering, Indiana University Purdue University Indianapolis, Indianapolis, IN 46202

*Corresponding author:

Julie Y. Ji

Department of Biomedical Engineering, Indiana University Purdue University Indianapolis

723 West Michigan Street, SL-220J, Indianapolis, IN 46202

Tel (317) 278-2275; Fax (317) 278-2455; Emails: jii@iupui.edu

Word Count: 6200

Figure Count: 2

This is the author's manuscript of the article published in final edited form as:

Jiang, Y., \& Ji, J. Y. (2018). Understanding lamin proteins and their roles in aging and cardiovascular diseases. Life Sciences. https://doi.org/10.1016/j.lfs.2018.09.026 


\begin{abstract}
The occurrence of cardiovascular diseases increases with age independent of other risk factors, and the percentage of senescent cells is significantly elevated in vascular cells at atherosclerotic sites. Patients with accelerated aging syndromes caused by mutant lamin A protein, a structural component in nuclear lamina, also share many similarities with normal aged people, including the propensity to develop atherosclerosis. Recent studies have revealed the accumulation of prelamin A in normal aged vascular cells, and that lamin A participated as a mechanosensitive molecule in regulating various cellular events. These findings suggest that the ectopic expression of mutant lamin A or lamin A precursor (prelamin A) not only causes defects in cell mechanics, but it also disturbs stress-induced mechanotransduction pathways involving lamin A, both of which may contribute to vascular dysregulation. This review summarizes the current understanding of how lamin proteins are involved in vascular cell during aging, with a particular focus on the effect of mechanical stresses from blood flow on nuclear lamina of endothelial cells. Related studies are clarifying the role of lamin A in the progression of atherosclerosis, which will aid in the development of potential therapies for those suffering from lamin A-associated accelerated aging syndromes.
\end{abstract}

Keywords: lamins, laminopathies, progerin, vascular aging, atherosclerosis 


\section{Introduction}

Cardiovascular disease (CVD) is among the leading causes of death in the United States [3]. The progression of CVD often initiates on the endothelium, which generally acts as the first barrier to separate pulsatile blood flow and the interstitium, and at the same time provides semi-permeability for solutes and fluids to establish vascular homeostasis [4-6]. Many vascular risk factors, such as hypercholesterolemia, hypertension and aging were found to disturb endothelial functions by activating endothelium, and that activation will promote the recruitment and differentiation of circulating monocytes at the local area, which is followed by smooth muscle cells (SMC) proliferation due to altered cell-cell communications. These cellular events accompanied with the overproduction of extracellular matrix (ECM) together accelerate the atheromatous plaque buildup [7]. This disease progression is known as atherosclerosis, and the plaque rupture can cause severe thrombosis and even death in cases such as stroke or heart attack [8].

Vascular cells experience mechanical stimuli (tangential shear stress and circumferential strain) that are generated by the pulsatile blood flow in vivo, as shown in Figure 1 [9]. These mechanical cues also play an important role in the progression of atherosclerosis. Previous studies have shown that the atherosclerosis formation sites are structure-specific, preferentially occurring at arterial curvatures and branching points. At these sites vascular cells experience oscillatory and low shear stress and increased cyclic strain [10-13]. These mechanical forces have been shown to constantly regulate vascular physiology and pathology. In endothelial cells, the actin stress fibers aligned along the direction of minimum substrate deformation, for example the direction oblique to applied uniaxial stretch in vitro [1419], probably through the activation of RhoA by Rho-guanine nucleotide exchange factors (Rho-GEF) [20]. After exposure to regular shear stress, the filaments reoriented along the flow direction in concomitance with remodeling of cell-substrate adhesion and cell-cell adherent junctions [21]. The combination of these two physiological mechanical forces can also produce a synergistic effect on actin remodeling patterns [22]. Moreover, the angle between the flow and cell axis could have differential effects on the activation of endothelial nitric oxide synthase (eNOS) and NF-KB (a proinflammatory transcription factor). These findings suggest the capacity of hemodynamic forces on remodeling the 
endothelial morphology in a force pattern dependent manner [23]. Selective gene expression and biochemical production in vascular cells can also be altered by distinct patterns of mechanical forces [2427]. Endothelial microRNAs (miRNAs) expression profile that is critical in regulating diverse cell events, including cell apoptosis and proliferation, was shown to depend on the shear stress pattern $[28,29]$. The production of endothelin-1, intercellular adhesion molecule 1 (ICAM-1), vascular cell adhesion molecule 1 (VCAM-1), E-selectin and NOS activity were also sensitive to shear pattern [14-16,18,19,30-32]. It is recently reported that the endothelial sensitivity to the flow can be regulated by non-canonical Wnt signaling [33]. Also, endothelial cells from different species or vascular beds can elicit differential oxidative responses and gene expression profiles toward mechanical forces [34-36]: Sung et al. reported that human aortic endothelial cells (HAEC) were more susceptible to fluid agitation, while HUVECs were more sensitive to cyclic strain in terms of the productions of reactive oxygen species (ROS) and cell adhesion molecules. These findings suggest that distinct mechanotransduction responses may exist in cells from different vascular origins [37].

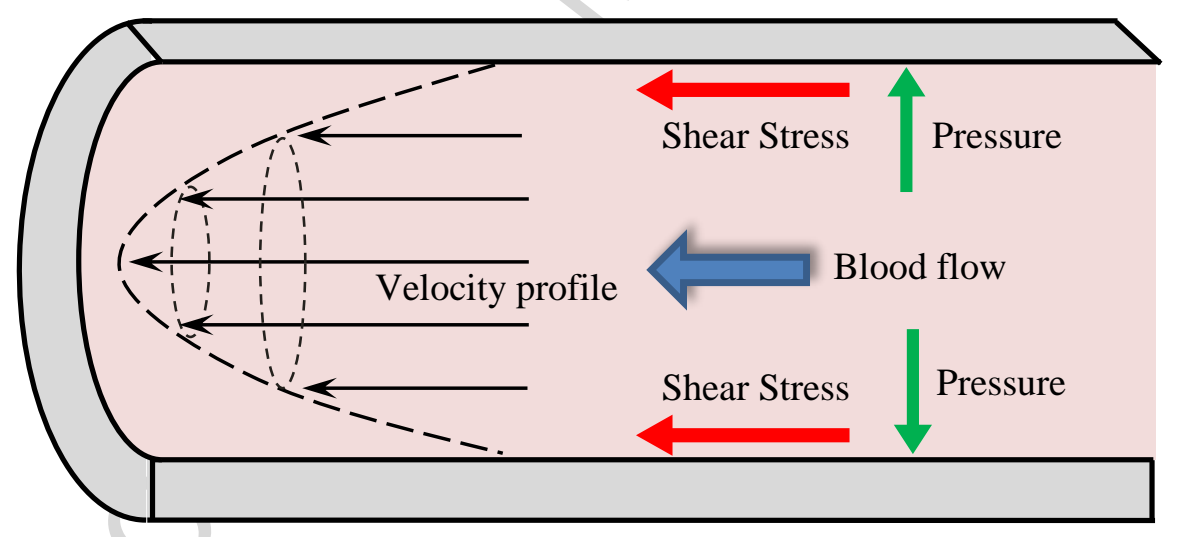

Figure 1. Illustrative image of main hemodynamic forces exerted on vascular wall. The viscous characteristic of blood flow induces non-uniform velocity profile within the blood vessel, which leads to the creation of shear stress parallel to the blood flow direction. The pulsatile blood flow also induces pressure changes onto the vessel wall [2], which on the other hand causes blood vessels to stretch circumferentially. Arrows denote the directions of blood flow and mechanical forces. Shear stress is tangential to vessel wall, while pressure is perpendicular.

The nucleus also plays a significant role in the mechanotransduction pathways by nucleocytoskeletal coupling via Linker of Nucleoskeleton and Cytoskeleton (LINC) complex [38], a mechanoresponsive protein complex associated within nuclear envelope, which contains SUN-domain proteins (SUN1 and 2) and KASH-domain proteins (Nesprin 1, 2, and 3) that bind to nuclear lamina and 
cytoskeleton, respectively [39]. Endogenous and exogenous forces as mechanical stimuli can trigger structural and compositional changes in nuclear envelope, and modulate gene expression and cell behaviors by activating many downstream regulators [40-42].

The integrity of nucleus is largely supported by nuclear lamina, a dynamic network that sits underneath the inner nuclear membrane composed of lamin polymers and lamin-associated proteins [43]. As the main contributor to nuclear mechanical properties, the lamina is crucial in preventing DNA damage during nuclear deformation and cell migration $[44,45]$, and its proximity to heterochromatin also enables its role in stabilizing spatial organization of genome [46]. It helps transfer forces to the tethered chromatin via coupling with LINC complex, and responds to changes in the ECM by mediating lamins expression, in a manner that helps maintain nuclear stiffness in various tissues $[47,48]$. The nuclear lamina is involved in various cellular events that determine cell fate, and defects in the lamina contribute to disease phenotypes and impaired mechanotransduction pathways.

Lamins are type $\mathrm{V}$ intermediate filament (IF) proteins recognized as a filamentous network at first glance [49]. Besides acting as a structural support for nuclei under mechanical stresses, lamins were also found to participate in DNA replication and repair, as well as in gene regulations through interactions with peripheral heterochromatins or mechanosensitive transcription factors (TFs) [50-53]. Recent findings have indicated correlations between mutated lamin forms and atherosclerosis during aging process, suggesting that lamin proteins may play a role in the development of atherosclerosis particularly during normal aging [54-56].

The lamins are classified into two subgroups in vertebrates: A-type lamins (encoded by LMNA gene) and B-type lamins (encoded by LMNB1 or LMNB2 gene) by different isoelectric values $[57,58]$. They both have the protein structures and domain interactions typical of intermediate filaments, and further associations of these lamin polymers eventually generate filaments with $10 \mathrm{~nm}$ in diameter in vitro [59-61]. Although evidence showed that A- and B-type lamins form distinct but interacting network in mammalian cells [62], the in vivo organization of lamins filaments remains unknown, and it is considered to be more heterogeneous than the observed woven-like network in Xenopus laevis oocyte, which only 
has B-type lamins [61]. Recent application of cryo-electron tomography (cryo-ET) on the lamina revealed its supramolecular organization: a $3.5 \mathrm{~nm}$ thick lamin filament meshwork with globular structures that represent the immunoglobulin-fold domains [63].

Within the lamin family, lamin A and lamin C are two major forms originated from LMNA gene by alternative splicing, and lamin B1 and lamin B2 are generated from LMNB1 and LMNB2, respectively [64]. All major lamins except lamin C possess a CAAX motif at the carboxyl-terminus after protein synthesis where the cysteine (C) is farnesylated by farnesyltransferase (FT) to facilitate the protein localization at the nuclear envelope (NE). After that, the aaX residues are hydrolyzed by farnesylated proteins-converting enzyme 2 (FACE2) on lamin B1 and B2, or by a zinc metallo-endoprotease (Zmpste24 or FACE-1) on prelamin A (the precursor for lamin A). The farnesylated cysteine at the end will be methylated afterwards, and all those steps will lead to the permanently farnesylated tails on B-type lamins. However, the additional removal of the last 15 amino acids (including the farnesylated cysteine) by Zmpste24 will occur on lamin A precursor before it proceeds to mature lamin A (Figure 2) [65].

Aside from the slight differences in post-translational steps, A- and B-type lamins were also found to perform differently in multiple cellular events. During mitosis, the nuclear lamina undergo disassembly, where A-type lamins diffused into the nucleoplasm at early prophase, while B-type lamins tended to be fragmented and concentrated near the mitotic spindle later during metaphase [66]. The reassembly timing for them also differed, indicating their diverse disassembly and reassembly mechanisms during cell division [67]. Furthermore, contributions of these lamins to nucleus mechanics are also distinct. While the ablation of lamin $\mathrm{A} / \mathrm{C}$ or lamin $\mathrm{A}$ resulted in a compromised nuclear shape and stiffness in fibroblasts, lamin B1-deficient cells had more nuclear blebs (membrane invaginations and protrusions) without discernible alternations in nuclear mechanics [68]. This discrepancy may be due to greater association and stabilization between A-type lamins and nuclear structural proteins, such as emerin, nesprins, and lamina-associated polypeptide 2 isoform alpha (LAP2 $\alpha$ ) [69-71]. 
A

$N$-terminal

$\alpha$-helical rod domain $\quad$ NLS Ig fold

C-terminal

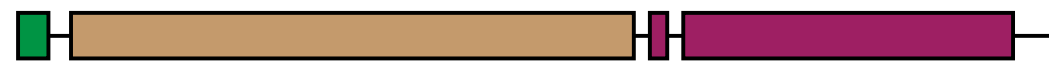

B

(a)

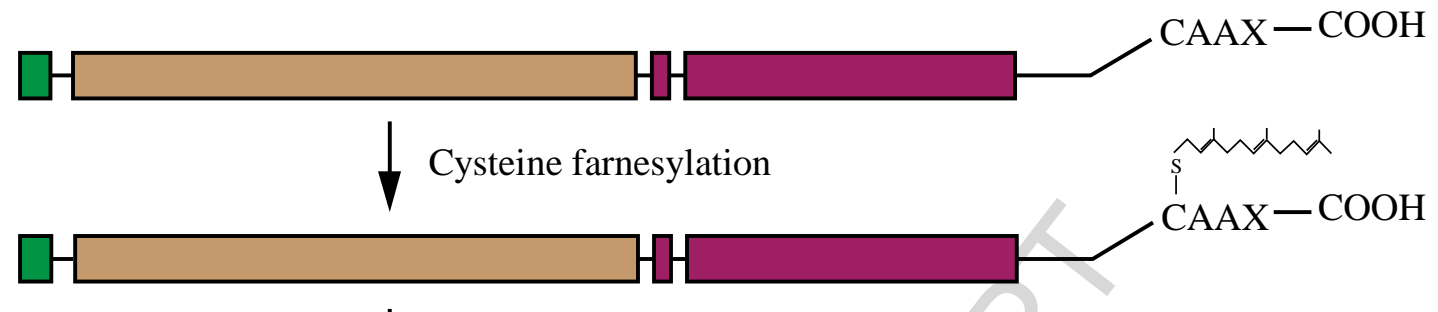

(b)

Hydrolysis of -aaX and carboxy methylation

(c)

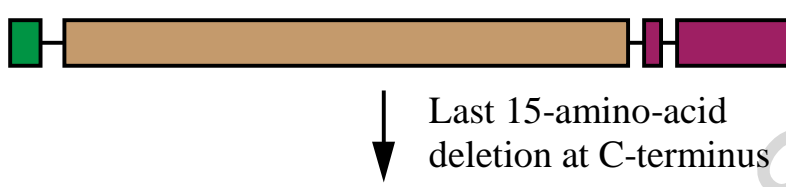

(d)

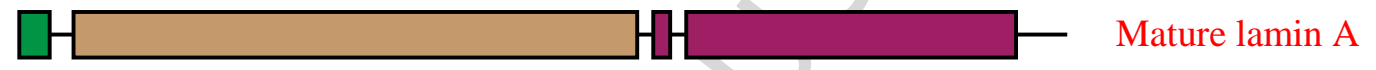

Figure 2. Schematic drawing of typical lamins structures (A), including a highly conserved $\alpha$-helix rod domain and a more divergent $\mathrm{N}$-terminal head and a C-terminal tail that contains NLS (nuclear localization signal that contains chromatin binding site) and Ig fold domain [1]. (B) Post-translational modifications in lamin proteins. Lamins after protein synthesis contain a complete-CAAX motif at C-terminus (a), and the cysteine in -CAAX motif is farnesylated by farnesyltransferase (b). The aaX residues are removed and the cysteine at the C-terminus is methylated (c). Mature lamin A will be generated after the last 15 amino acids being removed by ZMPSTE24 (a zinc metallo-endoprotease) (d).

In the past decades, more than 17 diseases have been reported to be associated with lamin gene mutations (mainly LMNA gene), and they are collectively named laminopathies [50]. Mutations involving LMNB1 and LMNB2 genes mostly cause disorders in neural development and degeneration, such as adult-onset autosomal dominant leukodystrophy (ADLD) and are often lethal [72-74]. The essential role that B-type lamins play in cell viability might explain the limited quantity of identified mutations in LMNB genes [75]. Conversely, over 500 missense LMNA mutations have been recognized that mainly affected the progeny of mesenchymal stem cells [76,77]. Patients with LMNA mutations often experienced multiple phenotypes, including defects in skeletal and cardiac muscles and loss of subcutaneous adipose tissue, such as in Emery-Dreifuss muscular dystrophy (EDMD) and Dunnigan-type familial partial lipodystrophy (FPLD) [78], as well as peripheral nerve dysfunctions, or disorders resembling aging syndromes in young patients (Hutchinson-Gilford progeria syndrome (HGPS)) [79]. 
Notably, the systematic premature aging syndromes (also known as progeroid syndromes) by missense mutations in LMNA, such as Atypical Werner syndrome (WS), Restrictive dermopathy (RD) and HGPS (also called Progeria) have attracted enormous attention given their correlations with the normal aging process. Multiple and overlapping phenotypes were identified in those diseases, including postnatal growth retardation, wrinkled skin, substantial hair loss [80]. While sequencing technique suggested genetic heterogeneity for MAD (Mandibuloacral Dysplasia) and Atypical WS [81], cells from HGPS patients were generally reported to have missense mutations in exon 11 on the LMNA gene. This creates a cryptic splice site responsible for the deletion of 50 amino acids near C-terminus on prelamin A, resulting in an absence of the recognition site for Zmpste24 to remove the final 15 -amino-acid at Cterminus. This lack of post-translational modification leads to the generation of a truncated prelamin A called progerin that has a permanently farnesylated tail. RD was reported to have an extra removal of 40 amino acids (totally 90) on prelamin A, which was believed to cause more severe outcomes than HGPS given its lethality in newborns $[82,83]$. Interestingly, similar phenotypes were also observed in patients or mouse models with mutations or deficiency in Zmpste24 gene [84].

Except for the resemblance in appearance between patients with progeroid syndromes (mainly HGPS) and elderly people, they also shared some similarities at the cellular level, including increased DNA damage, defects in DNA repair and shortened telomere length that significantly impaired cell proliferation capacity [85]. The accumulated DNA damage, as one of the hallmarks of normal aging, was often found in senescent cells $[86,87]$. Like elderly people, patients with progeriod syndrome were also prone to developing advanced atherosclerotic lesions, but the extensive loss of SMC in arteries and thickened adventitial fibrosis in veins were only observed in HGPS patients [88], and patients with laminopathies of premature aging did not exhibit all normal aging phenotypes [89]. However, the presence of truncated prelamin A in normal aged individuals (at a much lower level) as well as their high genomic instability, telomere attrition, epigenetic and metabolic alternations and cell senescence, particularly in cardiovascular system, still suggest lamin's role in promoting vascular aging [86,90-93]. While B-type lamins, especially lamin B1 were greatly lost in senescent cells [94], their deficiencies were 
recorded to mostly affect nervous system and brain function [72]. However, little evidence has been shown on the role of lamin B with vascular aging or nuclear mechanics [68], and the relationship between B-type lamins and cell senescence remain to be recognized. Related studies might shed more light on the discoveries on aging mechanisms and potential treatments for those patients suffered from accelerated aging diseases $[95,96]$.

\section{Roles of lamin $\mathrm{A} / \mathrm{C}$ in maintaining cell functionality}

Lamin A/C serves not only as structural components to support nuclear mechanics, but also interacts with many transcription factors and periphery heterochromatin to regulate gene expression [97,98]. Lamin A/C-associated disorders that affect the vascular system suggest that lamin A/C plays an important role in supporting cell mechanics and in signaling processes under mechanical forces, which may help to understand the underlying mechanisms of atherosclerotic lesion formation process $[77,88,99,100]$.

\subsection{Mechanical roles of Lamin A/C}

After incorporating into nuclear lamina, lamin $\mathrm{A} / \mathrm{C}$ forms a filamentous meshwork that modulates the dynamic mechanical properties of nucleus in accordance with the internal and external forces of the cell. This process is achieved not only by the physical connections among ECM, focal adhesions, cytoskeleton and nuclear envelope, but also by many mechanosensitive molecules that were recognized as transducing mechanosignals into nucleus to elicit changes in gene expression profiles [101]. For instance, ECM stiffness was found to be parallel to lamin A/C expression. The resulting post-translational modifications in lamin $\mathrm{A} / \mathrm{C}$ degradation and phosphorylation via coupling with myosin-IIA can lead to changes in the Serum Response Factor (SRF) pathway, which in turn regulates actomyosin expression [102]. This self-regulating mechanism involving lamin A/C allows cells to respond to its environmental stresses through changes in cell and nuclear mechanics. Since vascular cells exist in a mechanically active microenvironment, it was proposed that lamin $\mathrm{A} / \mathrm{C}$ is essential in modifying these cells behaviors in response to mechanical forces, and that may partially explain why the absence or mutations in lamin $\mathrm{A} / \mathrm{C}$ can induce the most abnormalities in cells that are under mechanical loadings [97]. 


\subsubsection{Lamin A/C Effects on Nuclear Mechanics}

Nuclear lamina was proposed to be a natively compressed and elastic network in static state, which can resist potential overloading force to prevent nuclear breakdown [103]. Different types of lamin might contribute to distinct mechanical properties of the nucleus. Observations from micropipette aspiration experiment revealed that B-type lamins contributed more to the nuclear elasticity, while A-type lamins acted to resist nuclear deformation as a viscous network [104,105].

While studies showed that lamin $\mathrm{A} / \mathrm{C}$ is homogeneously distributed at the nuclear periphery which delineates the nuclear shape, human dermal fibroblasts with silenced or mutated lamin A/C had significant nuclear defects in morphology, such as distorted nuclear periphery and nuclear blebs or invaginations [106]. Increased nuclear deformation was also observed in fibroblasts without lamin A/C present or with lamin mutations that can cause muscular defects. However, fibroblasts from FPLD patients showed normal nuclear stiffness [107]. And in HGPS skin fibroblasts that overexpressed progerin, the stiffness was reported to increase, as well as elevated mechanosensitivity [108]. Those findings are consistent with the varied laminopathy phenotypes by different de novo mutations of lamin $\mathrm{A} / \mathrm{C}$, indicating that distinct changes in nuclear mechanics can be induced by dissimilar amino acid substitutions on lamin A/C sequence, which could differentially affect the domains or higher order structures on lamin A/C, as well as their filamentous network within the lamina $[107,109]$.

Specifically, the increased nuclear stiffness in HGPS cells might be related to the pathological mechanism of progerin, which tended to aggregate at the nuclear periphery and hamper the deformation of nuclei toward mechanical forces $[108,110]$. The birefringent orientation of lamins found in HGPS cells may also contribute to the stiffened nuclei [111]. Other than the observed mechanical abnormalities in fibroblasts, the introduction of $\Delta 50$ lamin $\mathrm{A}$ in HeLa cells also disturbed their morphological responses to laminar shear stress. Similar nuclear defects were also reported in lamin A/C-deficient multinucleated myotubes, indicating the broad effects of lamin A/C on nuclear mechanics regardless of cell types $[68,112]$. While the treatment of farnesyltransferase inhibitors (FTI) has successfully rescued nuclear shape and the stiffness of HGPS fibroblasts, by removing the farnesylated tail, the cellular 
mechanosensitivity was not ameliorated [108]. Therefore, the recovery of stiffness by releasing progerin into nucleoplasm is not sufficient to help cells to resist external forces.

\subsubsection{Lamin A/C Effects on Whole Cell Mechanics}

Nuclear lamina has been shown to be associated with cytoplasmic actin filaments, microtubules and intermediate filaments through emerin and LINC complex [46]. Given the fact that the nucleus and cytoplasm are interlinked by physical connections [113], it is not surprising to observe the changes in whole cell mechanical properties when lamin $\mathrm{A} / \mathrm{C}$ was defective.

The deficiency of lamin A/C in mouse embryonic fibroblasts (MEFs) can lead to softened cytoplasm, compromised viscoelastic properties of the cytoskeleton, decreased cell polarization and migration rate $[114,115]$. Similar defects were observed in fibroblasts with mutant LMNA (Lmna ${ }^{\text {L530P/ }}$ L530P) that yield HGPS phenotypes in human [116]. These phenomena suggest that the dysfunctional lamin A/C can have adverse effects on cell mechanics. More specifically, silencing lamin A/C can greatly change cytoskeletal structures in MEFs and 3T3 cells, such as disturbed emerin and nesprin-3 localization, depolymerization of actin filaments at perinuclear regions, and abnormal absence or aggregation of vimentin [115]. However, in a mouse model with only lamin C expressed, the targeting of emerin to NE was reported to be normal [117]. This discrepancy might suggest the assistant role of lamin $\mathrm{C}$ in helping emerin at nuclear membrane. Mutations in LMNA responsible for muscular dystrophies were found to cause defective actin-dependent nuclear movement, while microtubule-mediated centrosome positioning was not interfered. On the other hand, in $\mathrm{LMNA}^{-/}$fibroblasts or those with LMNA mutations that cause HGPS or affect adipose tissues, emerin was mislocalized and related to that, disturbed microtubule-dependent cell polarization was observed [115,118]. These findings suggest distinct pathologies of laminopathies with different phenotypes [118].

Overall, evidence has shown that both nuclear and whole cell mechanics were weakened by mutated or deficient lamin $\mathrm{A} / \mathrm{C}$ with dysfunctions in various structural proteins, suggesting structuredependent complexity in lamin $\mathrm{A} / \mathrm{C}$ functions. These findings also highlight the necessity to distinguish and separate the effects brought by different mutated isoforms of lamin A/C. Moreover, the observation 
that releasing progerin from nuclear periphery did not completely recover cellular mechanosensitivity suggests that there might be additional pathogenic mechanisms for mutated lamin A with the farnesylated tail, besides its ability to disturb cell mechanics by permanently attaching to the lamina network.

\subsubsection{Lamin A/C Effects on Vasculature System and Endothelial Cells}

Although lamin A and lamin $\mathrm{C}$ are broadly present in many differentiated tissues, their expression levels were reported to scale with tissue elasticity that is largely determined by the surrounding ECM. In other words, stiffer tissue elicited more lamin A/C expression, while softer tissue had B-type lamins dominant [105]. Moreover, exclusive lamin C expression was observed in mouse central nervous system (cerebellum and cortex) except in capillary endothelial cells and the meninges. This is proposed to be linked to miR-9, a brain specific microRNA that was bound to prelamin A and downregulated its expression [105,119]. Therefore, it is critical to be aware of the distinct expression patterns and functions that lamin A/C might have in tissues possessing different stiffness. Arterial stiffness, on another hand, is associated with other factors, including endothelial signaling and VSMCs (vascular smooth muscle cells) tone that were also found to be modified by lamin A/C [120-122]. Given the associated mechanical defects due to the genetically mutated lamin A, it is worthwhile to analyze how lamin A/C modifies vascular cells in cell mechanics.

Many articles have addressed the vascular specific disorders brought by abnormal lamin A/C. LMNA knockout mice that exhibited dilated cardiomyopathy (DCM) had altered nuclear shape and disrupted connections between the nucleus and desmin (muscle-specific IF) in cardiomyocytes and skeletal muscle cells [123-125]. Several missense mutations identified in patients with DCM were also found to affect cardiac conduction system or heart muscle [126]. Reduced lamin A/C expression that was induced by hypertension promoted the hyperproliferation of VSMCs, indicating the ability of lamin A/C in preventing stretch-induced VSMC proliferation. The suppressions of lamin $\mathrm{A} / \mathrm{C}$ and nesprin expressions by low shear stress in primary rat aortic ECs also indicate the potential dysregulations of lamin A/C-related nucleo-cytoskeletal connections induced by abnormal mechanical stress in ECs [127]. These data suggest the mechanosensitive role of lamin A in vascular system. Further studies are needed to 
better understand the role of abnormal nuclear lamina in morphological remodeling processes of endothelial cells under flow.

\subsection{Signaling Roles of A-type Lamins}

Besides acting as a structural support to prevent the nucleus from breakdown, lamin $\mathrm{A} / \mathrm{C}$ also binds to various transcription factors and chromatins. They are present both at the nuclear periphery and throughout the nucleoplasm to form relatively stable structures in a dynamic equilibrium state, and interact with other nuclear molecules to mediate cell functions [128].

\subsubsection{Molecular Interactions of Lamin A/C}

A-type lamins associate with chromatins at lamina-associated domains (LADs) that repress gene expression in most cases, and they also interact with many transcription factors to regulate cell differentiation and proliferation [129-134]. Qi, et al. classified the primary functions of DNA segments that were bound to lamin A/C in VSMCs, and more than 35\% of the DNA was of the phosphoprotein category [135], indicating the role of lamin $\mathrm{A} / \mathrm{C}$ in post-translational modifications and signaling molecules regulations. Abnormal fragmentation of heterochromatin, changes in epigenetic marks and mislocalizations of transcription factors were also reported in cells with deficient or mutated LMNA gene, suggesting their critical roles in maintaining cell functionality through epigenetic regulations [136-139].

Multiple mechanisms are believed to contribute to these lamin A/C-associated gene regulations such as: providing the interaction platform for signaling molecules, hampering the import of transcription factor units to inhibit signal transduction, as well as regulating tissue-specific pathways by organizing periphery heterochromatin [140]. For example, megakaryoblastic leukaemia 1(MKL1), a transcription factor that regulates cell differentiation, migration and proliferation, failed to localize to nucleus in LMNA $^{-/}$and LMNA N195K mutant MEFs due to defective actin dynamics and loss of emerin expression [141]. Impaired Wnt signaling and reduced nuclear localization of lymphoid enhancer binding factor (LEF1) in a HGPS mouse model were observed to affect ECM synthesis [142]. Moreover, the critical position of lamin $\mathrm{A} / \mathrm{C}$ in nucleo-cytoskeletal coupling complex also facilitates their involvement in mechanotransduction pathways, for instance, the attenuated activation of NF- $\kappa \mathrm{B}$ signaling by biaxial 
cyclic strain in LMNA ${ }^{-/}$MEFs $[143,144]$. This finding suggests that mutation in or lack of lamin A/C can prompt not only changes in mechanical properties, but also in mechanotransduction pathways induced by external stimuli.

\subsubsection{Lamin A/C in Mechanosignaling Pathways Associated with Atherosclerosis}

Lamin $\mathrm{A} / \mathrm{C}$ has been shown to participate in many mechanotransduction pathways in vascular cells, and their mutations can led to vascular dysfunctions in DNA repair and inflammatory responses $[145,146]$. Studies have revealed their pivotal roles in many mechanosignaling pathways to maintain vascular homeostasis, and changes in applied mechanical forces can interfere with lamin $\mathrm{A} / \mathrm{C}$-associated regulations in vascular cells. For instance, A-type lamins and emerin were reported to suppress VSMC proliferation in vitro under normal cyclic strain (5\%), which was correlated with their associations with sequence-specific regions that enrich transcriptional factor motifs E2F1, E2F3, SP1 and Stat 1 [135]. Bovine aorta endothelial cells (BAEC) had defective shear stress-induced transcriptional activation of glucocorticoid response element (GRE) after silencing lamin A/C [147]. Low shear stress applied on primary aortic ECs resulted in increased proliferation and apoptosis in ECs, and the changes were shown to be induced by reduced lamin A/C expression and repressed activation of several TFs (Stat-1, Stat-3, Stat-5 and Stat-6) [127]. Those findings indicate the atheroprotective roles of lamin A/C in suppressing the stretch-induced VSMCs proliferation that can promote atherosclerotic lesion formation, as well as in participating shear stress-induced atheroprotective gene regulations in vascular ECs $[11,148]$. Moreover, the differential effects of lamin A expression on ECs and VSMCs in terms of low shear stress-induced secretary molecules PDGF-BB and TGF- $\beta$ productions also indicate the existence of cell type-specific response of A-type lamins in vascular system [149].

There are mainly two types of laminopathies involving atherosclerosis progression, FPLD2 and HGPS. Patients with FPLD2 that carry LMNA mutation at 482 codon often developed coronary heart disease in their adulthood, especially in women $[150,151]$. It was believed that metabolic disorders like insulin resistance contribute most to the premature atherosclerosis $[152,153]$, but a recent study revealed that the R482W LMNA mutation responsible for FPLD2 led to the cascade of endothelial dysfunctional 
events, including reduced NOS expression, activated proinflammatory molecules such as IL-6 and IL-8, and increased adhesion affinity with immune cells [154]. Similarly, the proinflammatory responses were also reported to be effected in HGPS ECs [7]. Combined with previous discussions of how hemodynamic forces regulate those endothelial functions in pathogenesis of atherosclerosis, it is suggested that the mutated lamin $\mathrm{A} / \mathrm{C}$ isoforms can potentially disturb the atheroprotective pathways, and promote the expressions of atheroprone genes in endothelial cells at arterial sites that are exposed to irregular mechanical stresses.

\section{Correlations Between Lamin A/C and Cellular Aging}

Lamin A/C expression seems to be regulated throughout development. For instance, evidence showed that lamin A/C did not appear until embryonic cell differentiation and the downregulation of several pluripotency genes like Tra-1-60 and SSEA-4 [155]. Their expression levels also varied in differentiated human tissues with differences in tissue stiffness $[105,156]$. Changes of lamin A/C over time can lead to many epigenetic changes, such as the induction of aberrant pathways that promote tumor cell migration and invasion in several types of cancers as well as in the aging process, which will be addressed in the following section [157].

\subsection{Lamin A/C in Aging Process and Progeriod Syndromes}

Aging refers to the natural process that an organism undergoes over time, which comes with declinations in body functions and increasing probability of developing diseases and death. Many mechanisms of aging have been proposed recently, including genomic instability, telomere attrition, epigenetic alternations, loss of proteostasis, deregulated nutrient sensing, mitochondrial dysfunction,cellular senescence, stem cell exhaustion, and altered intercellular communication [86,158]. Cell senescence is a state of arrested cells growth with altered gene expression profile. It is triggered either by telomere shortening (replicative senescence) or other damage signals before cells reach their limited lifespan (premature senescence) $[159,160]$. Recent studies in the structural and functional alternations of lamin $\mathrm{A} / \mathrm{C}$ in aging-related diseases revealed the similarities between the underlying mechanisms of laminopathies with that of aging pathologies, especially in nuclear architecture changes, 
chromatin and epigenetic modifications and activation of senescence pathways [161-163]. Some aging phenotypes such as cellular senescence and telomere dysfunction were induced by several laminopathies or diseases with abnormal Zmpste24 expression [164-167]. These abnormalities have motivated the research on how lamin A/C-related dysfunctions contribute to the normal aging process.

Many studies have addressed the role of progerin, the aberrant prelamin A isoform that causes HGPS, in contributing to accelerated aging. The expression of exogeneous progerin in normal fibroblast can induce replicative senescence and loss of telomeres, suggesting conserved progerin-dependent senescence pathways in normal cells [166]. In HGPS fibroblasts, progerin also accumulated in culture, accompanied with deteriorated nuclear shape and disorganized methylation pattern of histone 3 on lysine 9 (H3K9) that overlapped with the phenotypes found in aged cells [168]. Positive detection of $\Delta 150$ LMNA mRNA that was responsible for progerin synthesis was reported in normal fibroblasts, and the use of cryptic splicing site increased as the passage went up regardless of donors' age [169]. Meanwhile, the localization of wild type lamin $\mathrm{A} / \mathrm{C}$ was also altered in cells from old donors, where they were more accumulated at the nuclear envelope instead of present at both nuclear periphery and nucleoplasm as in young donor cells. Removal of progerin in cells from old individuals by the inhibition of the cryptic splicing site has rescued the nuclear defects in terms of the expression patterns of nuclear protein (HP1) and histone modifications (Tri-Me-K9H3) [170]. It is not yet known how progerin takes effects in normal aged cells at a very low expression level that did not change significantly over age (although the increasing trend was observed over in vitro culture) [171]. On the other hand, the downregulation of Zmpste24 gene due to increased sensitivity to oxidative stress during aging might be another cause for the prelamin A accumulation in aged cells [172]. Lack of Zmpste24 will interfere the prelamin A posttranslational modifications, and lamin A isoforms that possess the permanently farnesylated tail will be generated instead of the mature form $[173,174]$.

\subsection{Molecular Mechanisms of Lamin A/C-dependent Premature Aging}

Multiple but not mutually exclusive mechanisms were proposed about how lamin A/C participates in cell senescence. Some studies hypothesized that progerin production in HGPS cells was 
mediated by SRSF1 (serine-arginine rich splicing factor 1) and SRSF6, whose expressions were also agedependent $[175,176]$. Gene therapy to block the aberrant splicing site reversed the nuclear defects, and treated HGPS mice had elongated life span [177]. Another treatment by FTIs that inhibit prelaimin A farnesylation had rescued nuclear shape, and improved bone structure and vascular stiffness in both mouse models and patients with HGPS, as well as prevented SMC loss in mice model [178-183].

However, this treatment did not ameliorate DNA damage or reactivate checkpoint signaling pathways in HGPS and RD human fibroblasts. Other remnant problems of this treatment like the universal effects of FTI on farnesylation inhibition, and the retention of prelamin A at nucleoplasm still remain to be solved [184].

Another hypothesis is that, the prelamin A-associated cell aging is caused by telomere shortening that can lead to alternative splicing events in multiple genes including LMNA [171]. Aberrations in chromosomes and telomeres were observed after inducing progerin into normal human dermal fibroblasts [167]. The introduction of exogenous telomerase into HGPS fibroblasts can significantly enhance cell propagation ability and prevent senescence with unchanged progerin level over passages, probably by interacting with telomeres and repressing p53 and Rb tumor-repressor pathways [171]. The production of progerin was only seen in cells with short telomeres, but was not observed in telomore-independent premature senescence induced by sodium butyrate or a histone deacetylase inhibitor [171]. These findings suggest that progerin-induced senescence was specifically driven by telomere shortening. However, this hypothesis remains to be challenged as limited signals were tested in this study.

Besides telomere shortening, the extensive damage in DNA repair system and the changes in lamin A-associated regulating pathways were also reported to be potential mechanisms of cellular aging. Increased DNA damage, as well as the failure to respond to DNA damage and the hyperactivation of p53 tumor suppressor pathway, were observed in Zmpste24 ${ }^{-/-}$fibroblast, indicating dysregulated DNA repair in response to accumulated farnesylated prelamin A [163,185]. As a result of DNA damage in Zmpste24

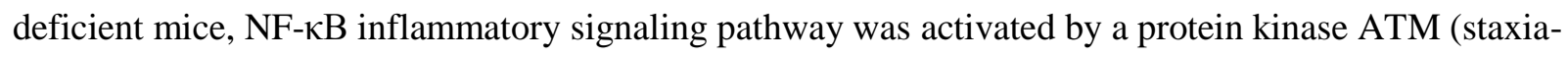
telangiectasia mutated) and its downstream factor NF- $\mathrm{B}$ essential modulator (NEMO). This pathway was 
involved in some progeroid phenotypes in those mice, since blocking the pathway greatly improved their biological characteristics [186-188]. However, the intact DNA repair pathways observed in a progeria mouse model with lamin A exon 9 deleted may indicate the trivial role of exon 9 region in maintaining genome stability [189]. Changes in epigenetic marks such as H3K27me3 and their causative heterochromatin reorganizations in human HGPS fibroblasts occurred prior to aberrations in nuclear shape [190]. Together with changes in histone methylation in HGPS and aging cells, these data suggest that general mechanisms of epigenetic control were shared by normal aging process and prelamin Ainduced premature aging diseases[91]. A more recent study showed that signaling pathway driven by a sequestered transcription factor NRF2 (nuclear factor (erythroid-derived)-like 2) was impaired in HGPS fibroblasts. It is a substrate for CAND1 gene with the capacity to prevent HGPS defects in vitro. NRF2 expression was closely associated with progerin expression, antioxidant gene activation, and cell survival [191]. Although reactivation of NRF2 pathway did not completely restore all progerin-induced defects, this finding disclosed one of the molecular mechanisms in progerin-induced cellular defects, and provided new insight into lamin-A associated aging process.

Furthermore, recent advances in stem cell research also indicate that regenerative dysfunctions may also contribute to premature aging [192,193]. The multiple roles that Notch signaling plays in vascular system may also be the link between progerin and vascular dysregulations. Notch signaling pathway was activated by ectopic progerin expression in human mesenchymal stem cells (hMSCs) [194]. The capacity of hMSCs to differentiate into adipocytes was largely compromised, which is consistent with the subcutaneous adipose tissue loss in HGPS patients [195].

Overall, efforts have been invested to explore the underlying mechanisms of prelamin A inducedsenescence. Confirmations of the role of prelamin A in in vivo aging and comparison of molecular mechanisms in normal and premature aging laminopathies are needed to further understand how immature lamin A contributes to the aging process.

\subsection{Lamin A/C in Vascular Aging}


As healthy individuals age, the risk of developing cardiovascular disease increases with age as a result of changes in in structure and functions of the vasculature [196-198]. Age-associated changes in vascular cells and ECM composition, such as elevated secretion of proinflammatory factors, increased invasive and migratory potentials, reduced production of vasodilators, as well as the changes in collagen and elastin components can lead to altered mechanical properties of vessel wall and inflammation [199,200]. For example, the stiffness and adhesion capacity of VSMCs were increased with aging [201], and the expression pattern of Sirt1, a deacetylase involving in multiple cellular events including antiinflammatory pathways in endothelial cells [202], was observed to be repressed in vascular aging [203]. Similar phenotypes were reported in prelamin A-induced progeroid syndromes, including characteristics of stiffened and calcified vessel wall, and activation of ICAM-1 and monocytes adhesion in ECs [204206]. Nevertheless, different phenotypes also existed between HGPS and aging individuals that were summarized by Brassard and colleagues [199], such as extensive atherosclerotic lesions, increased platelet count, prolonged prothrombin times, as well as the preserved flow-mediated dilation response in HGPS vessels, indicating dose-dependent effects of progerin in vascular system [207]. However, considering the similar vascular defects induced by prelamin A and physiological aging, the prelamin A-associated changes in vasculature may still be indicative of vascular aging.

Evidence of restrictive detection of progerin in vascular cells from a HGPS patient indicates the preference of progerin accumulation in vascular system [100], and the activations of inflammatory responses and cell senescence in vascular ECs from FPLD2 patients also suggest the onset of atherosclerosis [154]. Immunostaining in a progeria mouse model revealed a significant loss of SMCs in large arteries accompanied with replacement of proteoglycan (PG) and collagen, and it was followed by adventitial thickening and arterial wall calcification that were the main contributors to vascular stiffening [204,208]. Several progeria mouse models were created to study the mechanisms underlying prelamin Ainduced premature aging, but not all HGPS syndromes were recapitulated by these models [90], for instance, the observation of intimal thickening in HGPS patients was not reported in the mouse model 
[208], indicating the differences in progeria models across species, and the necessity of confirming the pathological findings in humans.

Similarly, prelamin A accumulation in normal aging vascular cells can also impair their functions and disturb the vascular remodeling process. The detection of endogenous prelamin A was reported in VSMCs either from aged individuals or by serial culture in vitro, and it was accompanied with the increased sensitivity towards differentiation prior to compromised proliferation capacity, suggesting that prelamin A accumulation precedes the onset of senescence [55]. Although this attenuation was believed to be initiated by the downregulation of Zmpste24 along with cellular senescence [209], their expressions were heterogeneous in advanced atherosclerotic lesions, indicating additional regulatory factors responsible for prelamin A accumulation [55]. ECs and endothelial progenitor cells with inhibited Zmpste24 activity overexpressed farnesylated prelamin A, and the inflammatory factor ICAM-1 was activated (but not VCAM-1 and E-selectin), and the adhesion of monocyte was enhanced in ECs. The regenerative capacity in endothelial progenitor cells was also impaired in terms of microvascular network generation, which indicates the exhaustion of endothelial stem cell pool [206], together with the observation of reduced migration potential in melanoma cells overexpressing $\Delta 50$ lamin A explain the low incident rate of cancer in progeria patients, since neovascularization is critical for cancer development and progression [210,211].

Besides the potential mechanisms discussed in the last section, altered cellular responses toward mechanical stresses were also closely linked to prelamin A-associated vascular disorders [199]. Cytoskeletal reorganizations, such as the reduced expressions of cytoskeletal proteins in SMCs under high shear stress in a progeria mouse model, were potentially responsible for the SMC loss in the medial layer of aorta during vasculopathy development [212]. Alternations in the mechanical properties of the vessel wall by prelamin A, such as vascular stiffening, were also critical in promoting vascular diseases [213]. One of the causes - ECM composition changes and the resulting proliferation arrest were observed in lamin A mutated fibroblasts, which is led by impaired Wnt signaling [142]. 
Overall, abnormalities in lamin $\mathrm{A} / \mathrm{C}$ have been suggested to cause premature aging phenotypes and influence vascular system functions. The observations of prelamin A accumulation in normal aged cells also indicate the pivotal roles it could play in promoting the aging process and vascular diseases. Further validations of how prelamin A participates in cellular senescence and vascular aging, as well as their associated pathways are needed to assist in developing therapeutic mechanisms for prelamin Ainduced premature aging diseases.

\section{Conclusion}

Previous studies have demonstrated that, the development of atherosclerosis was closely related to the hemodynamic forces that stimulate various responses in vascular cells. On the other hand, the abnormalities of LMNA gene expression were reported in either healthy aged cells or cells from patients with lamin A-associated aging syndromes. Considering the mechanosensitive roles that lamin A plays in vascular cells, it is important to investigate whether lamin A can mediate these stress-induced responses, which could be an underlying mechanism that contributes to the initiation of cardiovascular diseases.

Besides the advances in research that were discussed above, there are several remaining concerns to be addressed to fully understand the relationship between A-type lamins and atherosclerosis development during aging. For example, what are the functional differences between lamin A and lamin $\mathrm{C}$ in the mammalian cell aging process, and are they complementary to each other? How do senescent cells communicate with its surrounding non-senescent cells to achieve tissue-level changes? Ongoing research on these topics will help to explain the occurrence of laminopathies with accelerated aging syndrome, as well as the onset of vascular diseases with aging as the risk factor. 


\section{Acknowledgement}

This research did not receive any specific grant from funding agencies in the public, commercial, or notfor-profit sectors.

\section{Conflict of Interest}

The authors declare that there are no conflicts of interest. A signed Conflict of Interests Policy Form is submitted. 


\section{REFERENCES}

[1] Koster, S., D. A. Weitz, R. D. Goldman., et al., Intermediate filament mechanics in vitro and in the cell: from coiled coils to filaments, fibers and networks, Curr Opin Cell Biol. 32 (2015) 82-91.

[2] Estrada, R., G. A. Giridharan, M. D. Nguyen., et al., Endothelial cell culture model for replication of physiological profiles of pressure, flow, stretch, and shear stress in vitro, Anal Chem. 83 (2011) 3170-3177.

[3] Mozaffarian, D., E. J. Benjamin, A. S. Go., et al., Executive Summary: Heart Disease and Stroke Statistics-2016 Update A Report From the American Heart Association, Circulation. 133 (2016) 447-454.

[4] Mehta, D., A. B. Malik, Signaling mechanisms regulating endothelial permeability, Physiol Rev. 86 (2006) 279-367.

[5] Widmer, R. J., A. Lerman, Endothelial dysfunction and cardiovascular disease, Glob Cardiol Sci Pract. (2014) 43.

[6] Stevens, T., Y. Nakahashi, D. N. Cornfield., et al., Ca(2+)-inhibitable adenylyl cyclase modulates pulmonary artery endothelial cell cAMP content and barrier function, Proceedings of the National Academy of Sciences of the United States of America. 92 (1995) 2696-2700.

[7] Gimbrone, M. A., Jr., G. Garcia-Cardena, Endothelial Cell Dysfunction and the Pathobiology of Atherosclerosis, Circulation research. 118 (2016) 620-636.

[8] Bentzon, J. F., F. Otsuka, R. Virmani., et al., Mechanisms of plaque formation and rupture, Circulation research. 114 (2014) 1852-1866.

[9] Meza, D., The Effect of Combined Fluid Shear Stress and Cyclic Tensile Stretch on Vascular Endothelial Cells. State University of New York at Stony Brook, (2017).

[10] Baeyens, N., C. Bandyopadhyay, B. G. Coon., et al., Endothelial fluid shear stress sensing in vascular health and disease, The Journal of clinical investigation. 126 (2016) 821-828.

[11] Lehoux, S., E. A. Jones, Shear stress, arterial identity and atherosclerosis, Thrombosis and haemostasis. 115 (2016) 467-473.

[12] Chau, A. H., R. C. Chan, M. Shishkov., et al., Mechanical analysis of atherosclerotic plaques based on optical coherence tomography, Ann Biomed Eng. 32 (2004) 1494-1503.

[13] Kaazempur-Mofrad, M. R., H. F. Younis, S. Patel., et al., Cyclic strain in human carotid bifurcation and its potential correlation to atherogenesis: Idealized and anatomicallyrealistic models, Journal of Engineering Mathematics. 47 (2003) 299-314.

[14] Wang, J. H., P. Goldschmidt-Clermont, F. C. Yin, Contractility affects stress fiber remodeling and reorientation of endothelial cells subjected to cyclic mechanical stretching, Ann Biomed Eng. 28 (2000) 1165-1171.

[15] Iba, T., B. E. Sumpio, Morphological response of human endothelial cells subjected to cyclic strain in vitro, Microvasc Res. 42 (1991) 245-254.

[16] Naruse, K., T. Yamada, M. Sokabe, Involvement of SA channels in orienting response of cultured endothelial cells to cyclic stretch, Am J Physiol. 274 (1998) H1532-1538.

[17] Hayakawa, K., N. Sato, T. Obinata, Dynamic reorientation of cultured cells and stress fibers under mechanical stress from periodic stretching, Exp Cell Res. 268 (2001) 104-114.

[18] Sipkema, P., P. J. van der Linden, N. Westerhof., et al., Effect of cyclic axial stretch of rat arteries on endothelial cytoskeletal morphology and vascular reactivity, J Biomech. 36 (2003) 653-659. 
[19] Wang, J. H., P. Goldschmidt-Clermont, J. Wille., et al., Specificity of endothelial cell reorientation in response to cyclic mechanical stretching, J Biomech. 34 (2001) 15631572.

[20] Abiko, H., S. Fujiwara, K. Ohashi., et al., Rho guanine nucleotide exchange factors involved in cyclic-stretch-induced reorientation of vascular endothelial cells, Journal of cell science. 128 (2015) 1683-1695.

[21] Noria, S., F. Xu, S. McCue., et al., Assembly and reorientation of stress fibers drives morphological changes to endothelial cells exposed to shear stress, Am J Pathol. 164 (2004) 1211-1223.

[22] Zhao, S., A. Suciu, T. Ziegler., et al., Synergistic effects of fluid shear stress and cyclic circumferential stretch on vascular endothelial cell morphology and cytoskeleton, Arteriosclerosis, thrombosis, and vascular biology. 15 (1995) 1781-1786.

[23] Wang, C., B. M. Baker, C. S. Chen., et al., Endothelial cell sensing of flow direction, Arteriosclerosis, thrombosis, and vascular biology. 33 (2013) 2130-2136.

[24] Passerini, A. G., D. C. Polacek, C. Shi., et al., Coexisting proinflammatory and antioxidative endothelial transcription profiles in a disturbed flow region of the adult porcine aorta, Proceedings of the National Academy of Sciences of the United States of America. 101 (2004) 2482-2487.

[25] Dai, G., S. Vaughn, Y. Zhang., et al., Biomechanical forces in atherosclerosis-resistant vascular regions regulate endothelial redox balance via phosphoinositol 3-kinase/Aktdependent activation of Nrf2, Circulation research. 101 (2007) 723-733.

[26] Ding, Z., S. Liu, X. Wang., et al., Hemodynamic shear stress via ROS modulates PCSK9 expression in human vascular endothelial and smooth muscle cells and along the mouse aorta, Antioxidants \& redox signaling. 22 (2015) 760-771.

[27] Tang, X., Y. Liu, Q. Xiao., et al., Pathological cyclic strain promotes proliferation of vascular smooth muscle cells via the ACTH/ERK/STAT3 pathway, Journal of cellular biochemistry. (2018).

[28] Ni, C. W., H. Qiu, H. Jo, MicroRNA-663 upregulated by oscillatory shear stress plays a role in inflammatory response of endothelial cells, American journal of physiology. Heart and circulatory physiology. 300 (2011) H1762-1769.

[29] Weber, M., M. B. Baker, J. P. Moore., et al., MiR-21 is induced in endothelial cells by shear stress and modulates apoptosis and eNOS activity, Biochem Bioph Res Co. 393 (2010) 643-648.

[30] Ziegler, T., K. Bouzourene, V. J. Harrison., et al., Influence of oscillatory and unidirectional flow environments on the expression of endothelin and nitric oxide synthase in cultured endothelial cells, Arteriosclerosis, thrombosis, and vascular biology. 18 (1998) 686-692.

[31] Chappell, D. C., S. E. Varner, R. M. Nerem., et al., Oscillatory shear stress stimulates adhesion molecule expression in cultured human endothelium, Circulation research. 82 (1998) 532-539.

[32] Nagel, T., N. Resnick, W. J. Atkinson., et al., Shear stress selectively upregulates intercellular adhesion molecule-1 expression in cultured human vascular endothelial cells, The Journal of clinical investigation. 94 (1994) 885-891.

[33] Franco, C. A., M. L. Jones, M. O. Bernabeu., et al., Non-canonical Wnt signalling modulates the endothelial shear stress flow sensor in vascular remodelling, Elife. 5 (2016). 
[34] Du, W., I. Mills, B. E. Sumpio, Cyclic strain causes heterogeneous induction of transcription factors, AP-1, CRE binding protein and NF-kB in endothelial cells: Species and vascular bed diversity, Journal of Biomechanics. 28 (1995) 1485-1491.

[35] Wang, D. L., B. S. Wung, J. J. Cheng., et al., Cyclic strain-induced monocyte chemotactic protein-1 (MCP-1) gene expression in endothelial cells involves reactive oxygen species activation of AP-1., Faseb J. 11 (1997) 1667-1667.

[36] Busse, R., I. Fleming, Regulation of endothelium-derived vasoactive autacoid production by hemodynamic forces, Trends in Pharmacological Sciences. 24 (2003) 24-29.

[37] Sung, H. J., A. Yee, S. G. Eskin., et al., Cyclic strain and motion control produce opposite oxidative responses in two human endothelial cell types, Am J Physiol Cell Physiol. 293 (2007) C87-94.

[38] Tajik, A., Y. J. Zhang, F. X. Wei., et al., Transcription upregulation via force-induced direct stretching of chromatin, Nat Mater. 15 (2016) 1287-1296.

[39] Chang, W., H. J. Worman, G. G. Gundersen, Accessorizing and anchoring the LINC complex for multifunctionality, Journal of Cell Biology. 208 (2015) 11-22.

[40] Isermann, P., J. Lammerding, Nuclear mechanics and mechanotransduction in health and disease, Curr Biol. 23 (2013) R1113-1121.

[41] McGregor, A. L., C. R. Hsia, J. Lammerding, Squish and squeeze - the nucleus as a physical barrier during migration in confined environments, Curr Opin Cell Biol. 40 (2016) 32-40.

[42] Kirby, T. J., J. Lammerding, Emerging views of the nucleus as a cellular mechanosensor, Nat Cell Biol. 20 (2018) 373-381.

[43] Goldberg, M. W., J. Fiserova, I. Huttenlauch., et al., A new model for nuclear lamina organization, Biochem Soc T. 36 (2008) 1339-1343.

[44] Raab, M., M. Gentili, H. de Belly., et al., ESCRT III repairs nuclear envelope ruptures during cell migration to limit DNA damage and cell death, Science. 352 (2016) 359-362.

[45] Denais, C. M., R. M. Gilbert, P. Isermann., et al., Nuclear envelope rupture and repair during cancer cell migration, Science. 352 (2016) 353-358.

[46] Mattout, A., D. S. Cabianca, S. M. Gasser, Chromatin states and nuclear organization in development--a view from the nuclear lamina, Genome biology. 16 (2015) 174.

[47] Swift, J., D. E. Discher, The nuclear lamina is mechano-responsive to ECM elasticity in mature tissue, Journal of cell science. 127 (2014) 3005-3015.

[48] Majkut, S., P. C. D. P. Dingal, D. E. Discher, Stress Sensitivity and Mechanotransduction during Heart Development, Curr Biol. 24 (2014) R495-R501.

[49] Scheer, U., J. Kartenbeck, M. F. Trendelenburg., et al., Experimental disintegration of the nuclear envelope. Evidence for pore-connecting fibrils, The Journal of cell biology. 69 (1976) 1-18.

[50] Gruenbaum, Y., R. Foisner, Lamins: Nuclear Intermediate Filament Proteins with Fundamental Functions in Nuclear Mechanics and Genome Regulation, Annu Rev Biochem. 84 (2015) 131-164.

[51] Ho, C. Y., J. Lammerding, Lamins at a glance, Journal of cell science. 125 (2012) 20872093.

[52] Mislow, J. M., J. M. Holaska, M. S. Kim., et al., Nesprin-1alpha self-associates and binds directly to emerin and lamin A in vitro, FEBS letters. 525 (2002) 135-140.

[53] Lee, K. K., T. Haraguchi, R. S. Lee., et al., Distinct functional domains in emerin bind lamin A and DNA-bridging protein BAF, Journal of cell science. 114 (2001) 4567-4573. 
[54] Ghosh, S., Z. J. Zhou, Genetics of aging, progeria and lamin disorders, Curr Opin Genet Dev. 26 (2014) 41-46.

[55] Ragnauth, C. D., D. T. Warren, Y. Liu., et al., Prelamin A acts to accelerate smooth muscle cell senescence and is a novel biomarker of human vascular aging, Circulation. 121 (2010) 2200-2210.

[56] Garg, A., Gender differences in the prevalence of metabolic complications in familial partial lipodystrophy (Dunnigan variety), J Clin Endocr Metab. 85 (2000) 1776-1782.

[57] Krohne, G., R. Benavente, The nuclear lamins. A multigene family of proteins in evolution and differentiation, Exp Cell Res. 162 (1986) 1-10.

[58] Shelton, K. R., L. L. Higgins, D. L. Cochran., et al., Nuclear lamins of erythrocyte and liver, The Journal of biological chemistry. 255 (1980) 10978-10983.

[59] Kolb, T., K. Maass, M. Hergt., et al., Lamin A and lamin C form homodimers and coexist in higher complex forms both in the nucleoplasmic fraction and in the lamina of cultured human cells, Nucleus. 2 (2011) 425-433.

[60] Ben-Harush, K., N. Wiesel, D. Frenkiel-Krispin., et al., The supramolecular organization of the C. elegans nuclear lamin filament, Journal of molecular biology. 386 (2009) 13921402.

[61] Gruenbaum, Y., O. Medalia, Lamins: the structure and protein complexes, Curr Opin Cell Biol. 32 (2015) 7-12.

[62] Xie, W., A. Chojnowski, T. Boudier., et al., A-type Lamins Form Distinct Filamentous Networks with Differential Nuclear Pore Complex Associations, Curr Biol. 26 (2016) 2651-2658.

[63] Turgay, Y., M. Eibauer, A. E. Goldman., et al., The molecular architecture of lamins in somatic cells, Nature. 543 (2017) 261-264.

[64] Burke, B., C. L. Stewart, The nuclear lamins: flexibility in function, Nat Rev Mol Cell Biol. 14 (2013) 13-24.

[65] Casasola, A., D. Scalzo, V. Nandakumar., et al., Prelamin A processing, accumulation and distribution in normal cells and laminopathy disorders, Nucleus. 7 (2016) 84-102.

[66] Georgatos, S. D., A. Pyrpasopoulou, P. A. Theodoropoulos, Nuclear envelope breakdown in mammalian cells involves stepwise lamina disassembly and microtubule-drive deformation of the nuclear membrane, Journal of cell science. 110.17 (1997) 2129-2140.

[67] Moriuchi, T., M. Kuroda, F. Kusumoto., et al., Lamin A reassembly at the end of mitosis is regulated by its SUMO-interacting motif, Exp Cell Res. 342 (2016) 83-94.

[68] Lammerding, J., L. G. Fong, J. Y. Ji., et al., Lamins A and C but not lamin B1 regulate nuclear mechanics, J Biol Chem. 281 (2006) 25768-25780.

[69] Lattanzi, G., V. Cenni, S. Marmiroli., et al., Association of emerin with nuclear and cytoplasmic actin is regulated in differentiating myoblasts, Biochem Bioph Res Co. 303 (2003) 764-770.

[70] Al-Haboubi, T., D. K. Shumaker, J. Koser., et al., Distinct association of the nuclear pore protein Nup153 with A- and B-type lamins, Nucleus-Austin. 2 (2011) 500-509.

[71] Markiewicz, E., T. Dechat, R. Foisner., et al., Lamin A/C binding protein LAP2 alpha is required for nuclear anchorage of retinoblastoma protein, Mol Biol Cell. 13 (2002) 44014413.

[72] Coffinier, C., H. J. Jung, C. Nobumori., et al., Deficiencies in lamin B1 and lamin B2 cause neurodevelopmental defects and distinct nuclear shape abnormalities in neurons, Mol Biol Cell. 22 (2011) 4683-4693. 
[73] Padiath, Q. S., K. Saigoh, R. Schiffmann., et al., Lamin B1 duplications cause autosomal dominant leukodystrophy, Nat Genet. 38 (2006) 1114-1123.

[74] Lin, S. T., M. Y. Heng, L. J. Ptacek., et al., Regulation of Myelination in the Central Nervous System by Nuclear Lamin B1 and Non-coding RNAs, Transl Neurodegener. 3 (2014) 4.

[75] Hutchison, C. J., B-type lamins in health and disease, Semin Cell Dev Biol. 29 (2014) 158163.

[76] Gruenbaum, Y., R. Foisner, Lamins: nuclear intermediate filament proteins with fundamental functions in nuclear mechanics and genome regulation, Annu Rev Biochem. 84 (2015) 131-164.

[77] Zhang, J. Q., Q. Z. Lian, G. L. Zhu., et al., A Human iPSC Model of Hutchinson Gilford Progeria Reveals Vascular Smooth Muscle and Mesenchymal Stem Cell Defects, Cell Stem Cell. 8 (2011) 31-45.

[78] Yajima, R., T. Takahashi, Y. Higuchi., et al., Japanese case of Emery-Dreifuss muscular dystrophy with a novel LMNA missense mutation, Neurol Clin Neurosci. 4 (2016) 124-+.

[79] Zhang, S., K. Zhang, M. Jiang., et al., Hutchinson-Gilford progeria syndrome with scleroderma-like skin changes due to a homozygous missense LMNA mutation, J Eur Acad Dermatol. 30 (2016) 463-465.

[80] Dorado, B., V. Andres, A-type lamins and cardiovascular disease in premature aging syndromes, Curr Opin Cell Biol. 46 (2017) 17-25.

[81] Simha, V., A. K. Agarwal, E. A. Oral., et al., Genetic and phenotypic heterogeneity in patients with mandibuloacral dysplasia-associated lipodystrophy, J Clin Endocr Metab. 88 (2003) 2821-2824.

[82] Schreiber, K. H., B. K. Kennedy, When Lamins Go Bad: Nuclear Structure and Disease, Cell. 152 (2013) 1365-1375.

[83] Navarro, C. L., A. De Sandre-Giovannoli, R. Bernard., et al., Lamin A and ZMPSTE24 (FACE-1) defects cause nuclear disorganization and identify restrictive dermopathy as a lethal neonatal laminopathy, Human molecular genetics. 13 (2004) 2493-2503.

[84] Wang, Y. X., U. Lichter-Konecki, K. Anyane-Yeboa., et al., A mutation abolishing the ZMPSTE24 cleavage site in prelamin A causes a progeroid disorder, Journal of cell science. 129 (2016) 1975-1980.

[85] Gonzalo, S., R. Kreienkamp, P. Askjaer, Hutchinson-Gilford Progeria Syndrome: A premature aging disease caused by LMNA gene mutations, Ageing Res Rev. 33 (2017) 18-29.

[86] Lopez-Otin, C., M. A. Blasco, L. Partridge., et al., The hallmarks of aging, Cell. 153 (2013) 1194-1217.

[87] Ribezzo, F., Y. Shiloh, B. Schumacher, Systemic DNA damage responses in aging and diseases, Semin Cancer Biol. 37-38 (2016) 26-35.

[88] Olive, M., I. Harten, R. Mitchell., et al., Cardiovascular pathology in Hutchinson-Gilford progeria: correlation with the vascular pathology of aging, Arteriosclerosis, thrombosis, and vascular biology. 30 (2010) 2301-2309.

[89] Dreesen, O., C. L. Stewart, Accelerated aging syndromes, are they relevant to normal human aging?, Aging (Albany NY). 3 (2011) 889-895.

[90] Hamczyk, M. R., L. del Campo, V. Andres, Aging in the Cardiovascular System: Lessons from Hutchinson-Gilford Progeria Syndrome, Annu Rev Physiol. 80 (2018) 27-48. 
[91] Kubben, N., T. Misteli, Shared molecular and cellular mechanisms of premature ageing and ageing-associated diseases, Nat Rev Mol Cell Bio. 18 (2017) 595-609.

[92] Olive, M., I. Harten, R. Mitchell., et al., Cardiovascular Pathology in Hutchinson-Gilford Progeria: Correlation With the Vascular Pathology of Aging, Arterioscl Throm Vas. 30 (2010) 2301-U2636.

[93] Tran, J. R., H. Y. Chen, X. B. Zheng., et al., Lamin in inflammation and aging, Curr Opin Cell Biol. 40 (2016) 124-130.

[94] Freund, A., R. M. Laberge, M. Demaria., et al., Lamin B1 loss is a senescence-associated biomarker, Mol Biol Cell. 23 (2012) 2066-2075.

[95] Cao, K., J. J. Graziotto, C. D. Blair., et al., Rapamycin reverses cellular phenotypes and enhances mutant protein clearance in Hutchinson-Gilford progeria syndrome cells, Sci Transl Med. 3 (2011) 89ra58.

[96] Harhouri, K., C. Navarro, C. Baquerre., et al., Antisense-Based Progerin Downregulation in HGPS-Like Patients' Cells, Cells-Basel. 5 (2016).

[97] Dahl, K. N., A. J. Ribeiro, J. Lammerding, Nuclear shape, mechanics, and mechanotransduction, Circulation research. 102 (2008) 1307-1318.

[98] Bronshtein, I., E. Kepten, I. Kanter., et al., Loss of lamin A function increases chromatin dynamics in the nuclear interior, Nat Commun. 6 (2015).

[99] Quiros-Gonzalez, I., P. Roman-Garcia, C. Alonso-Montes., et al., Lamin A is involved in the development of vascular calcification induced by chronic kidney failure and phosphorus load, Bone. 84 (2016) 160-168.

[100] McClintock, D., L. B. Gordon, K. Djabali, Hutchinson-Gilford progeria mutant lamin A primarily targets human vascular cells as detected by an anti-Lamin A G608G antibody, Proceedings of the National Academy of Sciences of the United States of America. 103 (2006) 2154-2159.

[101] Davidson, P. M., J. Lammerding, Broken nuclei--lamins, nuclear mechanics, and disease, Trends Cell Biol. 24 (2014) 247-256.

[102] Buxboim, A., J. Swift, J. Irianto., et al., Matrix elasticity regulates lamin-A,C phosphorylation and turnover with feedback to actomyosin, Curr Biol. 24 (2014) 19091917.

[103] Dahl, K. N., S. M. Kahn, K. L. Wilson., et al., The nuclear envelope lamina network has elasticity and a compressibility limit suggestive of a molecular shock absorber, Journal of cell science. 117 (2004) 4779-4786.

[104] Shimi, T., K. Pfleghaar, S. Kojima., et al., The A- and B-type nuclear lamin networks: microdomains involved in chromatin organization and transcription, Genes Dev. 22 (2008) 3409-3421.

[105] Swift, J., I. L. Ivanovska, A. Buxboim., et al., Nuclear Lamin-A Scales with Tissue Stiffness and Enhances Matrix-Directed Differentiation, Science. 341 (2013).

[106] De Vos, W. H., F. Houben, R. A. Hoebe., et al., Increased plasticity of the nuclear envelope and hypermobility of telomeres due to the loss of A-type lamins, Biochim Biophys Acta. 1800 (2010) 448-458.

[107] Zwerger, M., D. E. Jaalouk, M. L. Lombardi., et al., Myopathic lamin mutations impair nuclear stability in cells and tissue and disrupt nucleo-cytoskeletal coupling, Human molecular genetics. 22 (2013) 2335-2349. 
[108] Verstraeten, V. L., J. Y. Ji, K. S. Cummings., et al., Increased mechanosensitivity and nuclear stiffness in Hutchinson-Gilford progeria cells: effects of farnesyltransferase inhibitors, Aging Cell. 7 (2008) 383-393.

[109] de Leeuw, R., Y. Gruenbaum, O. Medalia, Nuclear Lamins: Thin Filaments with Major Functions, Trends Cell Biol. 28 (2018) 34-45.

[110] Kalinowski, A., P. N. Yaron, Z. Qin., et al., Interfacial binding and aggregation of lamin A tail domains associated with Hutchinson-Gilford progeria syndrome, Biophys Chem. 195 (2014) 43-48.

[111] Dahl, K. N., P. Scaffidi, M. F. Islam., et al., Distinct structural and mechanical properties of the nuclear lamina in Hutchinson-Gilford progeria syndrome, Proceedings of the National Academy of Sciences of the United States of America. 103 (2006) 1027110276.

[112] Philip, J. T., K. N. Dahl, Nuclear mechanotransduction: response of the lamina to extracellular stress with implications in aging, Journal of biomechanics. 41 (2008) 31643170.

[113] Guilluy, C., K. Burridge, Nuclear mechanotransduction: Forcing the nucleus to respond, Nucleus. 6 (2015) 19-22.

[114] Lee, J. S., C. M. Hale, P. Panorchan., et al., Nuclear lamin A/C deficiency induces defects in cell mechanics, polarization, and migration, Biophys J. 93 (2007) 2542-2552.

[115] Houben, F., C. H. Willems, I. L. Declercq., et al., Disturbed nuclear orientation and cellular migration in A-type lamin deficient cells, Biochim Biophys Acta. 1793 (2009) 312-324.

[116] Hale, C. M., A. L. Shrestha, S. B. Khatau., et al., Dysfunctional connections between the nucleus and the actin and microtubule networks in laminopathic models, Biophys J. 95 (2008) 5462-5475.

[117] Fong, L. G., J. K. Ng, J. Lammerding., et al., Prelamin A and lamin A appear to be dispensable in the nuclear lamina, Journal of Clinical Investigation. 116 (2006) 743-752.

[118] Folker, E. S., C. Ostlund, G. W. G. Luxton., et al., Lamin A variants that cause striated muscle disease are defective in anchoring transmembrane actin-associated nuclear lines for nuclear movement, Proceedings of the National Academy of Sciences of the United States of America. 108 (2011) 131-136.

[119] Jung, H. J., C. Coffinier, Y. Choe., et al., Regulation of prelamin A but not lamin C by miR-9, a brain-specific microRNA, Proceedings of the National Academy of Sciences of the United States of America. 109 (2012) E423-E431.

[120] Zieman, S. J., V. Melenovsky, D. A. Kass, Mechanisms, pathophysiology, and therapy of arterial stiffness, Arteriosclerosis, thrombosis, and vascular biology. 25 (2005) 932-943.

[121] Figueiredo, V. N., J. C. Yugar-Toledo, L. C. Martins., et al., Vascular stiffness and endothelial dysfunction: Correlations at different levels of blood pressure, Blood Pressure. 21 (2012) 31-38.

[122] Sehgel, N. L., Y. Zhu, Z. Sun., et al., Increased vascular smooth muscle cell stiffness: a novel mechanism for aortic stiffness in hypertension, Am J Physiol-Heart C. 305 (2013) H1281-H1287.

[123] Verga, L., M. Concardi, A. Pilotto., et al., Loss of lamin A/C expression revealed by immuno-electron microscopy in dilated cardiomyopathy with atrioventricular block caused by LMNA gene defects, Virchows Arch. 443 (2003) 664-671. 
[124] Chandar, S., L. S. Yeo, C. Leimena., et al., Effects of mechanical stress and carvedilol in lamin A/C-deficient dilated cardiomyopathy, Circulation research. 106 (2010) 573-582.

[125] Nikolova, V., C. Leimena, A. C. McMahon., et al., Defects in nuclear structure and function promote dilated cardiomyopathy in lamin A/C-deficient mice, The Journal of clinical investigation. 113 (2004) 357-369.

[126] Quarta, G., P. Syrris, M. Ashworth., et al., Mutations in the Lamin A/C gene mimic arrhythmogenic right ventricular cardiomyopathy, Eur Heart J. 33 (2012) 1128-1136.

[127] Han, Y., L. Wang, Q. P. Yao., et al., Nuclear envelope proteins Nesprin2 and LaminA regulate proliferation and apoptosis of vascular endothelial cells in response to shear stress, Biochim Biophys Acta. 1853 (2015) 1165-1173.

[128] Goldman, R. D., S. A. Adam, A. E. Goldman., et al., The nuclear lamins are major determinants of nuclear architecture, Febs Open Bio. 8 (2018) 72-72.

[129] Gibbs-Seymour, I., E. Markiewicz, S. Bekker-Jensen., et al., Lamin A/C-dependent interaction with 53BP1 promotes cellular responses to DNA damage, Aging Cell. 14 (2015) 162-169.

[130] Gesson, K., P. Rescheneder, M. P. Skoruppa., et al., A-type lamins bind both hetero- and euchromatin, the latter being regulated by lamina-associated polypeptide 2 alpha, Genome Res. 26 (2016) 462-473.

[131] Ruchaud, S., N. Korfali, P. Villa., et al., Caspase-6 gene disruption reveals a requirement for lamin A cleavage in apoptotic chromatin condensation, EMBO J. 21 (2002) 19671977.

[132] Lund, E., A. R. Oldenburg, E. Delbarre., et al., Lamin A/C-promoter interactions specify chromatin state-dependent transcription outcomes, Genome Res. 23 (2013) 1580-1589.

[133] Ivorra, C., M. Kubicek, J. M. Gonzalez., et al., A mechanism of AP-1 suppression through interaction of c-Fos with lamin A/C, Genes Dev. 20 (2006) 307-320.

[134] Solovei, I., A. S. Wang, K. Thanisch., et al., LBR and lamin A/C sequentially tether peripheral heterochromatin and inversely regulate differentiation, Cell. 152 (2013) 584598.

[135] Qi, Y. X., Q. P. Yao, K. Huang., et al., Nuclear envelope proteins modulate proliferation of vascular smooth muscle cells during cyclic stretch application, Proceedings of the National Academy of Sciences of the United States of America. 113 (2016) 5293-5298.

[136] Bruston, F., E. Delbarre, C. Ostlund., et al., Loss of a DNA binding site within the tail of prelamin A contributes to altered heterochromatin anchorage by progerin, FEBS letters. 584 (2010) 2999-3004.

[137] McCord, R. P., A. Nazario-Toole, H. Zhang., et al., Correlated alterations in genome organization, histone methylation, and DNA-lamin A/C interactions in HutchinsonGilford progeria syndrome, Genome Res. 23 (2013) 260-269.

[138] Cesarini, E., C. Mozzetta, F. Marullo., et al., Lamin A/C sustains PcG protein architecture, maintaining transcriptional repression at target genes, Journal of Cell Biology. 211 (2015) 533-551.

[139] Gonzalez-Granado, J. M., C. Silvestre-Roig, V. Rocha-Perugini., et al., Nuclear envelope lamin-A couples actin dynamics with immunological synapse architecture and T cell activation, Sci Signal. 7 (2014) ra37.

[140] Osmanagic-Myers, S., T. Dechat, R. Foisner, Lamins at the crossroads of mechanosignaling, Genes Dev. 29 (2015) 225-237. 
[141] Ho, C. Y., D. E. Jaalouk, M. K. Vartiainen., et al., Lamin A/C and emerin regulate MKL1SRF activity by modulating actin dynamics, Nature. 497 (2013) 507-511.

[142] Hernandez, L., K. J. Roux, E. S. M. Wong., et al., Functional Coupling between the Extracellular Matrix and Nuclear Lamina by Wnt Signaling in Progeria, Dev Cell. 19 (2010) 413-425.

[143] Lammerding, J., P. C. Schulze, T. Takahashi., et al., Lamin A/C deficiency causes defective nuclear mechanics and mechanotransduction, The Journal of clinical investigation. 113 (2004) 370-378.

[144] Kim, J. K., D. H. Kim, Lamin A/C Guided Nuclear Mechanotransduction, Biophys J. 114 (2018) 651a-651a.

[145] Afonso, P., M. Auclair, F. Boccara., et al., LMNA mutations resulting in lipodystrophy and HIV protease inhibitors trigger vascular smooth muscle cell senescence and calcification: Role of ZMPSTE24 downregulation, Atherosclerosis. 245 (2016) 200-211.

[146] Del Campo, L., M. R. Hamczyk, V. Andres., et al., Mechanisms of vascular aging: What can we learn from Hutchinson-Gilford progeria syndrome?, Clinica e investigacion en arteriosclerosis : publicacion oficial de la Sociedad Espanola de Arteriosclerosis. 30 (2018) 120-132.

[147] Nayebosadri, A., J. Y. Ji, Endothelial nuclear lamina is not required for glucocorticoid receptor nuclear import but does affect receptor-mediated transcription activation, Am J Physiol Cell Physiol. 305 (2013) C309-322.

[148] Bennett, M. R., S. Sinha, G. K. Owens, Vascular Smooth Muscle Cells in Atherosclerosis, Circulation research. 118 (2016) 692-702.

[149] Qi, Y. X., J. Jiang, X. H. Jiang., et al., PDGF-BB and TGF-\{beta 11 on cross-talk between endothelial and smooth muscle cells in vascular remodeling induced by low shear stress, Proceedings of the National Academy of Sciences of the United States of America. 108 (2011) 1908-1913.

[150] Eriksson, B. M. H., F. S. Collins, L. B. Gordon., et al., LMNA gene and its involvement in Hutchinson-Gilford Progeria Syndrome (HGPS) and arteriosclerosis. Google Patents, (2007).

[151] Guillin-Amarelle, C., A. Fernandez-Pombo, S. Sanchez-Iglesias., et al., Lipodystrophic laminopathies: Diagnostic clues, Nucleus. 9 (2018) 249-260.

[152] Palmer, N. D., R. D. Stevens, P. A. Antinozzi., et al., Metabolomic profile associated with insulin resistance and conversion to diabetes in the Insulin Resistance Atherosclerosis Study, The Journal of clinical endocrinology and metabolism. 100 (2015) E463-468.

[153] Hughes, T. M., S. Craft, L. D. Baker., et al., Changes in metabolic risk factors over 10 years and their associations with late-life cognitive performance: The Multi-Ethnic Study of Atherosclerosis, Alzheimer's \& Dementia: Diagnosis, Assessment \& Disease Monitoring. 8 (2017) 18-25.

[154] Bidault, G., M. Garcia, M. C. Vantyghem., et al., Lipodystrophy-linked LMNA p.R482W mutation induces clinical early atherosclerosis and in vitro endothelial dysfunction, Arterioscler Thromb Vasc Biol. 33 (2013) 2162-2171.

[155] Constantinescu, D., H. L. Gray, P. J. Sammak., et al., Lamin A/C expression is a marker of mouse and human embryonic stem cell differentiation, Stem Cells. 24 (2006) 177-185.

[156] Buxboim, A., J. Irianto, J. Swift., et al., Coordinated increase of nuclear tension and laminA with matrix stiffness outcompetes lamin-B receptor that favors soft tissue phenotypes, Mol Biol Cell. 28 (2017) 3333-3348. 
[157] Kong, L., G. Schafer, H. Bu., et al., Lamin A/C protein is overexpressed in tissue-invading prostate cancer and promotes prostate cancer cell growth, migration and invasion through the PI3K/AKT/PTEN pathway, Carcinogenesis. 33 (2012) 751-759.

[158] Farr, J. N., M. Almeida, The Spectrum of Fundamental Basic Science Discoveries Contributing to Organismal Aging, Journal of bone and mineral research : the official journal of the American Society for Bone and Mineral Research. (2018).

[159] van Deursen, J. M., The role of senescent cells in ageing, Nature. 509 (2014) 439-446.

[160] Childs, B. G., M. Durik, D. J. Baker., et al., Cellular senescence in aging and age-related disease: from mechanisms to therapy, Nat Med. 21 (2015) 1424-1435.

[161] Phillip, J. M., I. Aifuwa, J. Walston., et al., The Mechanobiology of Aging, Annual review of biomedical engineering. 17 (2015) 113-141.

[162] Moiseeva, O., F. Lessard, M. Acevedo-Aquino., et al., Mutant lamin A links prophase to a p53 independent senescence program, Cell cycle. 14 (2015) 2408-2421.

[163] Varela, I., J. Cadinanos, A. M. Pendas., et al., Accelerated ageing in mice deficient in Zmpste24 protease is linked to p53 signalling activation, Nature. 437 (2005) 564-568.

[164] von Muhlinen, N., I. Horikawa, F. Alam., et al., p53 isoforms regulate premature aging in human cells, Oncogene. 37 (2018) 2379-2393.

[165] Vidak, S., R. Foisner, Molecular insights into the premature aging disease progeria, Histochem Cell Biol. 145 (2016) 401-417.

[166] Huang, S., R. A. Risques, G. M. Martin., et al., Accelerated telomere shortening and replicative senescence in human fibroblasts overexpressing mutant and wild-type lamin A, Exp Cell Res. 314 (2008) 82-91.

[167] Benson, E. K., S. W. Lee, S. A. Aaronson, Role of progerin-induced telomere dysfunction in HGPS premature cellular senescence, J Cell Sci. 123 (2010) 2605-2612.

[168] Columbaro, M., C. Capanni, E. Mattioli., et al., Rescue of heterochromatin organization in Hutchinson-Gilford progeria by drug treatment, Cellular and molecular life sciences : CMLS. 62 (2005) 2669-2678.

[169] Scaffidi, P., T. Misteli, Lamin A-dependent nuclear defects in human aging, Science. 312 (2006) 1059-1063.

[170] Scaffidi, P., T. Misteli, Reversal of the cellular phenotype in the premature aging disease Hutchinson-Gilford progeria syndrome, Nat Med. 11 (2005) 440-445.

[171] Cao, K., C. D. Blair, D. A. Faddah., et al., Progerin and telomere dysfunction collaborate to trigger cellular senescence in normal human fibroblasts, The Journal of clinical investigation. 121 (2011) 2833-2844.

[172] Hernandez-Vallejo, S. J., C. Beaupere, J. Larghero., et al., HIV protease inhibitors induce senescence and alter osteoblastic potential of human bone marrow mesenchymal stem cells: beneficial effect of pravastatin, Aging Cell. 12 (2013) 955-965.

[173] Moiseeva, O., S. Lopes-Paciencia, G. Huot., et al., Permanent farnesylation of lamin A mutants linked to progeria impairs its phosphorylation at serine 22 during interphase, Aging-Us. 8 (2016) 366-381.

[174] Yu, K. R., K. S. Kang, Aging-related genes in mesenchymal stem cells: a mini-review, Gerontology. 59 (2013) 557-563.

[175] Holly, A. C., D. Melzer, L. C. Pilling., et al., Changes in splicing factor expression are associated with advancing age in man, Mech Ageing Dev. 134 (2013) 356-366. 
[176] Lopez-Mejia, I. C., V. Vautrot, M. De Toledo., et al., A conserved splicing mechanism of the LMNA gene controls premature aging, Human molecular genetics. 20 (2011) 45404555.

[177] Osorio, F. G., C. L. Navarro, J. Cadinanos., et al., Splicing-directed therapy in a new mouse model of human accelerated aging, Sci Transl Med. 3 (2011) 106ra107.

[178] Capell, B. C., M. R. Erdos, J. P. Madigan., et al., Inhibiting farnesylation of progerin prevents the characteristic nuclear blebbing of Hutchinson-Gilford progeria syndrome, Proceedings of the National Academy of Sciences of the United States of America. 102 (2005) 12879-12884.

[179] Bikkul, M. U., C. S. Clements, L. S. Godwin., et al., Farnesyltransferase inhibitor and rapamycin correct aberrant genome organisation and decrease DNA damage respectively, in Hutchinson-Gilford progeria syndrome fibroblasts, Biogerontology. (2018).

[180] Gordon, L. B., M. E. Kleinman, D. T. Miller., et al., Clinical trial of a farnesyltransferase inhibitor in children with Hutchinson-Gilford progeria syndrome, Proceedings of the National Academy of Sciences of the United States of America. 109 (2012) 1666616671.

[181] Fong, L. G., D. Frost, M. Meta., et al., A protein farnesyltransferase inhibitor ameliorates disease in a mouse model of progeria, Science. 311 (2006) 1621-1623.

[182] Mehta, I. S., C. H. Eskiw, H. D. Arican., et al., Farnesyltransferase inhibitor treatment restores chromosome territory positions and active chromosome dynamics in HutchinsonGilford progeria syndrome cells, Genome biology. 12 (2011) R74.

[183] Capell, B. C., M. Olive, M. R. Erdos., et al., A farnesyltransferase inhibitor prevents both the onset and late progression of cardiovascular disease in a progeria mouse model, Proceedings of the National Academy of Sciences of the United States of America. 105 (2008) 15902-15907.

[184] Adam, S. A., V. Butin-Israeli, M. M. Cleland., et al., Disruption of lamin B1 and lamin B2 processing and localization by farnesyltransferase inhibitors, Nucleus. 4 (2013) 142-150.

[185] Gonzalo, S., R. Kreienkamp, DNA repair defects and genome instability in HutchinsonGilford Progeria Syndrome, Curr Opin Cell Biol. 34 (2015) 75-83.

[186] Zhao, J., X. S. Li, S. McGowan., et al., NF-kappa B Activation with Aging: Characterization and Therapeutic Inhibition, Methods Mol Biol. 1280 (2015) 543-557.

[187] Osorio, F. G., C. Barcena, C. Soria-Valles., et al., Nuclear lamina defects cause ATMdependent NF-kappaB activation and link accelerated aging to a systemic inflammatory response, Genes Dev. 26 (2012) 2311-2324.

[188] Miyamoto, S., Nuclear initiated NF-kappaB signaling: NEMO and ATM take center stage, Cell Res. 21 (2011) 116-130.

[189] Das, A., D. A. Grotsky, M. A. Neumann., et al., Lamin A Deltaexon9 mutation leads to telomere and chromatin defects but not genomic instability, Nucleus. 4 (2013) 410-419.

[190] Arancio, W., G. Pizzolanti, S. I. Genovese., et al., Epigenetic Involvement in HutchinsonGilford Progeria Syndrome: A Mini-Review, Gerontology. 60 (2014) 197-203.

[191] Kubben, N., W. Zhang, L. Wang., et al., Repression of the Antioxidant NRF2 Pathway in Premature Aging, Cell. 165 (2016) 1361-1374.

[192] Wu, Z. M., W. Q. Zhang, M. S. Song., et al., Differential stem cell aging kinetics in Hutchinson-Gilford progeria syndrome and Werner syndrome, Protein Cell. 9 (2018) 333-350. 
[193] Brunauer, R., B. K. Kennedy, Progeria accelerates adult stem cell aging, Science. 348 (2015) 1093-1094.

[194] Perepelina, K., R. Dmitrieva, E. Ignatieva., et al., Lamin A/C mutation associated with lipodystrophy influences adipogenic differentiation of stem cells through interaction with Notch signaling, Biochemistry and Cell Biology. 96 (2018) 342-348.

[195] Kang, S. M., M. H. Yoon, B. J. Park, Laminopathies; Mutations on single gene and various human genetic diseases, Bmb Rep. 51 (2018) 327-337.

[196] North, B. J., D. A. Sinclair, The Intersection Between Aging and Cardiovascular Disease, Circulation research. 110 (2012) 1097-1108.

[197] Donato, A. J., R. G. Morgan, A. E. Walker., et al., Cellular and molecular biology of aging endothelial cells, J Mol Cell Cardiol. 89 (2015) 122-135.

[198] Sun, Z. J., Aging, Arterial Stiffness, and Hypertension, Hypertension. 65 (2015) 252-256.

[199] Brassard, J. A., N. Fekete, A. Garnier., et al., Hutchinson-Gilford progeria syndrome as a model for vascular aging, Biogerontology. 17 (2016) 129-145.

[200] Guzik, T. J., R. M. Touyz, Oxidative Stress, Inflammation, and Vascular Aging in Hypertension, Hypertension. 70 (2017) 660-667.

[201] Sehgel, N. L., Z. Sun, Z. K. Hong., et al., Augmented Vascular Smooth Muscle Cell Stiffness and Adhesion When Hypertension Is Superimposed on Aging, Hypertension. 65 (2015) 370-377.

[202] Kitada, M., Y. Ogura, D. Koya, The protective role of Sirt1 in vascular tissue: its relationship to vascular aging and atherosclerosis, Aging-Us. 8 (2016) 2290-2307.

[203] Donato, A. J., K. A. Magerko, B. R. Lawson., et al., SIRT-1 and vascular endothelial dysfunction with ageing in mice and humans, J Physiol-London. 589 (2011) 4545-4554.

[204] Gerhard-Herman, M., L. B. Smoot, N. Wake., et al., Mechanisms of premature vascular aging in children with Hutchinson-Gilford progeria syndrome, Hypertension. 59 (2012) 92-97.

[205] Minamino, T., I. Komuro, Vascular aging: insights from studies on cellular senescence, stem cell aging, and progeroid syndromes, Nat Clin Pract Cardiovasc Med. 5 (2008) 637648.

[206] Bonello-Palot, N., S. Simoncini, S. Robert., et al., Prelamin A accumulation in endothelial cells induces premature senescence and functional impairment, Atherosclerosis. 237 (2014) 45-52.

[207] Merideth, M. A., L. B. Gordon, S. Clauss., et al., Phenotype and course of HutchinsonGilford progeria syndrome, New Engl J Med. 358 (2008) 592-604.

[208] Varga, R., M. Eriksson, M. R. Erdos., et al., Progressive vascular smooth muscle cell defects in a mouse model of Hutchinson-Gilford progeria syndrome, Proceedings of the National Academy of Sciences of the United States of America. 103 (2006) 3250-3255.

[209] Yu, K. R., S. Lee, J. W. Jung., et al., MicroRNA-141-3p plays a role in human mesenchymal stem cell aging by directly targeting ZMPSTE24, Journal of cell science. 126 (2013) 5422-5431.

[210] Lin, C. C., W. L. Huang, W. C. Su, Lung cancer cell derived extracellular vesicles carrying DNAs, RNAs and proteins that may trigger oncogenic signals and promote angiogenesis, Cancer Res. 76 (2016).

[211] Zheng, L. F., X. M. Li, Y. Gu., et al., The 3'UTR of the pseudogene CYP4Z2P promotes tumor angiogenesis in breast cancer by acting as a ceRNA for CYP4Z1, Breast Cancer Res Tr. 150 (2015) 105-118. 
[212] Song, M. J., H. San, S. A. Anderson., et al., Shear stress-induced mechanotransduction protein deregulation and vasculopathy in a mouse model of progeria, Stem Cell Res Ther. 5 (2014).

[213] Kohn, J. C., M. C. Lampi, C. A. Reinhart-King, Age-related vascular stiffening: causes and consequences, Front Genet. 6 (2015). 